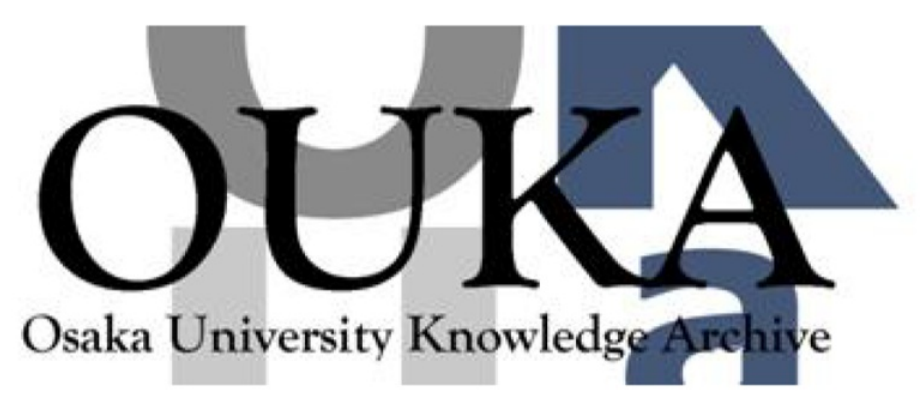

\begin{tabular}{|c|l|}
\hline Title & $\begin{array}{l}\text { An Energy-Efficient Broadcast Scheme for } \\
\text { Multihop Wireless Ad Hoc Networks Using } \\
\text { Variable-Range Transmission Power }\end{array}$ \\
\hline Author(s) & $\begin{array}{l}\text { Wong, TheinLai; Tsuchiya, Tatsuhiro; Kikuno, } \\
\text { Tohru }\end{array}$ \\
\hline Citation & $\begin{array}{l}\text { IEICE transactions on information and systems. } \\
\text { E90-D(3) p. 680-p. } 684\end{array}$ \\
\hline Issue Date & $2007-03-01$ \\
\hline oaire:version & VoR \\
\hline URL & https://hdl. handle. net/11094/27246 \\
\hline rights & $\begin{array}{l}\text { Copyright } \odot 2007 \text { The Institute of Electronics, } \\
\text { Information and Communication Engineers }\end{array}$ \\
\hline Note & \\
\hline
\end{tabular}

Osaka University Knowledge Archive : OUKA

https://ir. Library. osaka-u. ac. jp/

Osaka University 


\title{
LETTER \\ An Energy-Efficient Broadcast Scheme for Multihop Wireless Ad Hoc Networks Using Variable-Range Transmission Power
}

\author{
TheinLai WONG ${ }^{\dagger \text { a) }}$, Nonmember, Tatsuhiro TSUCHIYA ${ }^{\dagger}$, Member, and Tohru KIKUNO ${ }^{\dagger}$, Fellow
}

\begin{abstract}
SUMMARY This letter proposes a broadcast scheme for use in ad hoc networks using variable-range transmission power. Preserving energy and ensuring a high delivery ratio of broadcast packets are crucial tasks for broadcasting in ad hoc networks. Using individual broadcast relaying nodes to dynamically vary the transmission range can help saving power and reduce interference during communication. We analyzed the performance of the proposed scheme and compared it to other prevalent broadcast schemes for wireless ad hoc networks based on common-range transmission power.

key words: ad hoc network, broadcast, variable-range transmission, energy-efficient
\end{abstract}

\section{Introduction}

\subsection{Research Background}

Ad hoc networks are a special type of wireless network in which a collection of nodes with wireless interfaces form a temporary network, without the aid of any established infrastructure or centralized administration. The applications of ad hoc networks range from civilian applications to disaster recovery and military applications.

In a multi-hop ad hoc network, broadcasting is an elementary operation to support numerous applications. For example, broadcasting is commonly used in route discovery in ad hoc networks. Research indicates that naively broadcasting by flooding, where all of the nodes relay the received message, may cause serious message redundancy, contention, and collisions in the network. Collectively, we refer to the problems associated with flooding as the broadcast storm problem (see [1]). Furthermore, excessive redundant message relay will cause unnecessary power consumption in ad hoc networks.

\subsection{Research Objective}

In order to alleviate the broadcast storm problem, it is essential to limit the relaying nodes by using local information. Preserving energy in ad hoc networks is critical. Therefore, it is necessary to reduce power consumption. This can be achieved by transmitting packets in a shorter range, since the energy required to transmit a packet increases at least

Manuscript received July 25, 2006.

Manuscript revised October 20, 2006.

${ }^{\dagger}$ The authors are with the Graduate School of Information Science and Technology, Osaka University, Suita-shi, 565-0871 Japan.

a) E-mail: wong-t@ist.osaka-u.ac.jp DOI: 10.1093/ietisy/e90-d.3.680 quadratically with distance. However, shortening communication links is achieved at the expense of network connectivity. Therefore, in order to obtain good broadcast performance, it is important to select correct relaying nodes and to set an appropriate transmission power for each individual relaying node.

In this letter, we propose a scheme for broadcast in ad hoc networks using variable-range transmission power. We refer to this new scheme as the Variable-Range Broadcast Protocol (VRBP).

\section{Related Work}

Several approaches have been proposed to reduce the overall number of rebroadcast nodes. These broadcasting algorithms can be categorized mainly into probability-based methods and neighbor-knowledge-based methods (see [2]).

The probabilistic approach [1], [3] normally provides a simpler implementation, but it cannot guarantee full delivery.

In neighbor-knowledge-based methods, a node selects relaying nodes through a distributed process based on information of neighbors, which is obtained through periodic Hello messages.

Neighbor-knowledge-based methods can be further divided into self-pruning and neighbor designating methods. In self-pruning methods [4]-[8], each node makes its local decision as to whether to forward the received packet. In neighbor designating methods [9], [10], whether a node must forward the received packet is determined by its neighbors. A number of neighbor designating methods were compared by Williams and Camp [11].

In the protocols discussed above, the nodes always use the default maximum power level for transmission. We refer to these methods as common-range methods. In commonrange methods, such as SBA [7], AHBP [10], and MPR [9], energy efficiency can be achieved by setting an appropriate transmission range. The power level must be chosen statically. In other words, each node does not estimate the distance of its neighbors and does not take the distance information into consideration during the selection of relaying nodes (in the cases of AHBP and MPR), or when deciding whether to relay the received packet (in the case of SBA).

Several methods that employ variable transmission range have been proposed to make broadcasting energy efficient, and these methods can be categorized as either global [12] or local schemes [13], [14]. Global schemes such 
as [12] utilize global information that reflects the state of the network. Each node must know the distance between each pair of nodes and the transmission power needed for the pair to communicate. In contrast, localized schemes utilize only the information of nodes in the neighborhood, which can be obtained through periodic Hello message exchange, thereby reducing the latency incurred in the broadcast.

In [13], the distance information of each 1-hop neighbor must be included in the Hello message in order to provide sufficient information that is used in the relaying nodes selection process, and this incurs increased message overhead. Introducing shorter hops within 1-hop neighbors [14] may result in a longer latency for message delivery and a higher ratio of relaying nodes. In the proposed scheme, only 1-hop neighbors and the furthest distance among these 1hop neighbors are included in the Hello message in order to reduce the message overhead. Moreover, no intermediate relaying node is used to relay messages to other 1-hop neighbors, as in [14]. As a result, no additional message delivery latency is incurred, and the ratio of relaying nodes is lower.

\section{Energy-Efficient Broadcast Scheme}

In this section, we describe the proposed broadcast scheme, VRBP, in detail. Variable-Range Transmission Power is a neighbor designating scheme. Upon receiving the broadcast message, a node will check the message to see if it is designated as a Broadcast Relaying Node (BRN). In every received broadcast message, a BRN list and updated route information (the hops from the source to the previous sender) are included in the header of the message by the previous sender. A BRN must rebroadcast the received message. If a node is a designated BRN, it will select a new set of BRNs from its 1-hop neighbors based on information regarding its 2-hop neighbors and a number of selection criteria, which will be discussed later in this section. Finally, the node forwards the broadcast message with the BRNs and updated route information piggy-backed, using a tuned-down transmission power level that reaches its farthest BRN. The overall power consumption, which is the prime concern in the present study, can be reduced because each BRN does not transmit the broadcast message at its default maximum power.

\subsection{Neighbor Information Maintenance}

In order to select the new BRNs, a node must have knowledge of its 2-hop neighbors. This information can be obtained by exchanging Hello messages.

We assume that each node has a default maximum transmission power and Hello messages are sent at this maximum transmission power. One-hop neighbors are those neighbors that are within the corresponding maximum transmission range. The Hello message includes the information of the IDs of 1-hop neighbors and the furthest distance of its 1-hop neighbors. Each node calculates the distance from the sender each time a packet (data or Hello) is received. The distance between two communication nodes can be estimated by measuring the received signal strength [13].

Information of old neighbors is periodically deleted after a predetermined time in order to avoid out-of-date information.

\subsection{Relaying Node Selection and Transmission Range Set- ting}

A BRN calculates a new set of BRNs at the time of packet retransmission as follows:

1) Find the neighbors that have already received the same packet from the previous sender. These neighbors are excluded from the BRN selection described below. The BRN list and route information included in the header by the previous sender will be used to identify the 1-hop neighbors and 2-hop neighbors that have already received the same packet.

2) Find all 2-hop neighbors that can only be reached by one 1-hop neighbor. Assign this 1-hop neighbor as the BRN.

3) Check whether all 2-hop neighbors are covered. If not all 2-hop neighbors are covered, from the remaining 1hop neighbors not yet in the BRN list, find the one that would cover the most remaining 2-hop neighbors. When there are multiple nodes of this type, choose the one that has the shortest distance. For the case in which the distances to the nodes under consideration are all shorter than the longest distance for the nodes in the current BRN list, choose the node that requires a shorter transmission range to reach all of its 2-hop neighbors.

4) Repeat step 3 until all 2-hop neighbors are covered.

5) If there exists a 1-hop neighbor that has no 2-hop neighbor and is not located within the coverage range for the current farthest BRN in the BRN list, assign this node as a BRN. If there are multiple nodes of this type, assign only the node that has the farthest distance among these nodes as the BRN. The rest of the nodes are guaranteed to be covered because the final transmission range is set to reach the farthest BRN in the BRN set.

After the completion of BRN set selection, the transmission power level will be set to the power level needed in order to reach the farthest BRN in the BRN set constructed.

\section{Performance Evaluation}

In this section, we present the simulation results obtained by using the proposed broadcast algorithm. We conducted extensive simulations on various network conditions. The simulations were performed using network simulator 2 (NS2) [15].

We compared the proposed algorithm with simple flooding, SBA, and AHBP. SBA and AHBP have been proven in [11] to perform well in various scenarios compared to other schemes, such as counter-based schemes and 
Table 1 Parameter settings in the simulations.

\begin{tabular}{|l|c|}
\hline Simulation Parameter & Value \\
\hline \hline Network area & $500 \times 500$ meters \\
\hline Maximum transmission range & 100 meters \\
\hline Data packet size & 512 bytes \\
\hline Node max IFQ length & 50 \\
\hline MAC layer & IEEE 802.11 \\
\hline Hello message interval & 1 second \\
\hline Simulation time & 100 seconds \\
\hline Number of trials & 10 \\
\hline$c_{1}$ & $5.35 \mathrm{e}-7$ \\
\hline$c_{2}$ & $7.25 \mathrm{e}-11$ \\
\hline$d_{c}$ & 86.14 meters \\
\hline
\end{tabular}

location-based schemes.

Table 1 shows the parameter settings common to all of the scenarios investigated herein. In the present simulation, we consider only the power consumption due to data reception and data transmission. The power consumed for data reception is set to a constant ( 0.0035 watts), and the maximum power consumed (at maximum transmission range) for data transmission is set to 0.00725 watts. The transmission power is calculated based on the two-ray ground reflection model in NS2. In the two-ray reflection model, the relationship between transmission power, $P_{t}$, and distance, $d$, can be summarized as follows:

$$
P_{t}= \begin{cases}c_{1} d^{2}, & \text { if } d<d_{c} \\ c_{2} d^{4}, & \text { if } d \geq d_{c}\end{cases}
$$

In Eq. (1), $c_{1}$ and $c_{2}$ are constants and $d_{c}$ is the crossover distance in the two-ray ground reflection model. More detailed descriptions on the two-ray ground reflection model can be found in [15]. Generally, the transmission power drops drastically with the decrease in transmission range.

\subsection{Study 1: Congested Network}

In Study 1, we investigate the performance of the proposed variable-range broadcast scheme, VRBP, compared to other selected common-range schemes by varying the traffic load. The number of nodes is set to 100 .

Congestion can be produced by increasing the packet size, increasing the frequency of packet origination, or both. In this study, we chose to fix the packet size and varied the packet origination rate. Each new packet was originated by a source node randomly chosen from the network nodes to create a random traffic pattern.

Figure 1 shows that each scheme suffers as the network becomes more congested. Note that shortening the transmission range does not affect the delivery ratio, even for the congested network. The ratio of retransmission is also as low as that of AHBP (see Fig. 2). This is because VRBP does not incur any additional hops and each node only tunes down the transmission range needed from the default maximum range (100 meters) to the transmission range needed to reach its farthest BRN.

In Fig. 3, the total power consumption by VRBP and the other common-range schemes are plotted with respect

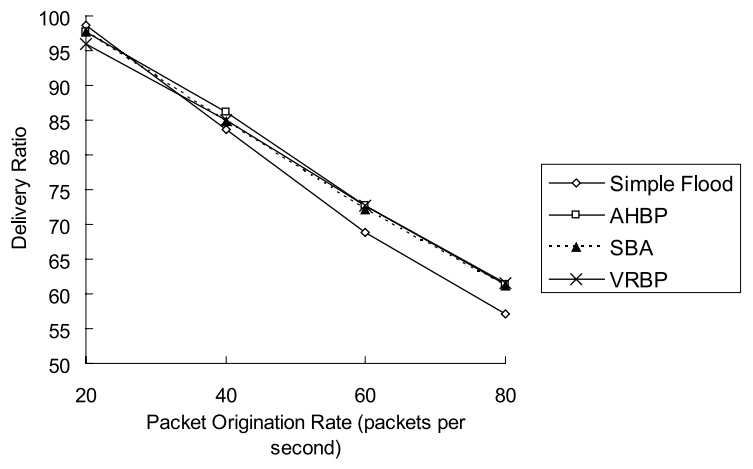

Fig. 1 Sensitivity of delivery ratio to traffic load.

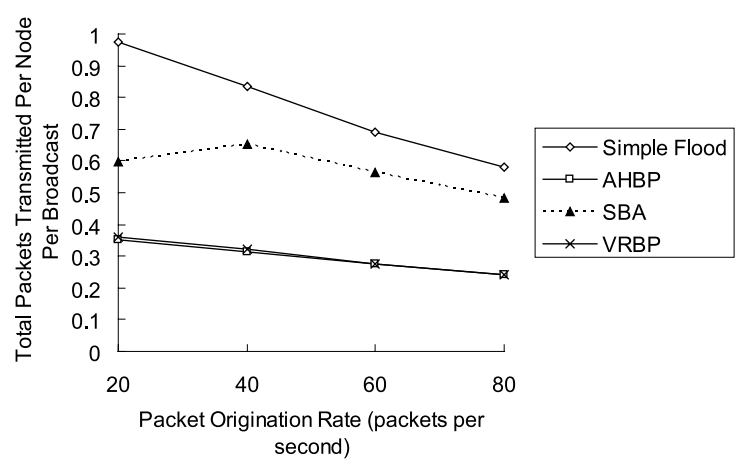

Fig. 2 Relationship between the ratio of rebroadcast nodes and the traffic load.

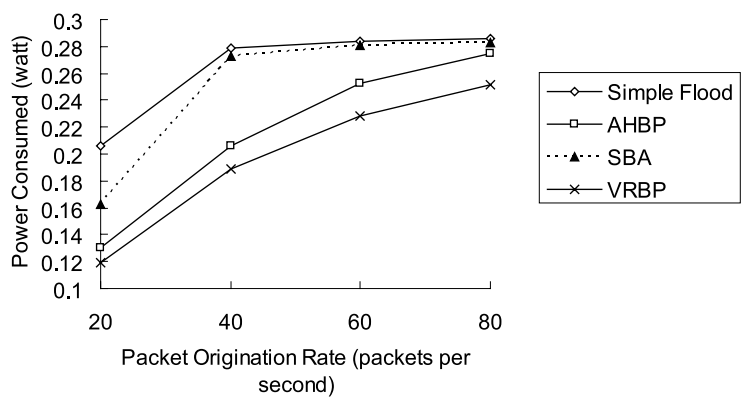

Fig. 3 Power consumption.

to various packet origination rates. Clearly, VRBP has a lower power consumption. Nevertheless, the percentage of power savings for VRBP decreases when the network becomes more congested.

Figure 4 shows the end-to-end delay of the proposed algorithm for various packet origination rates. The end-toend delay is the time it takes for the last node to receive a given packet. All schemes, except VRBP, experience a drastic increase in end-to-end delay as the traffic load increases. This can be explained by the improvement of the channel capacity when the interference is reduced.

\subsection{Study 2: Network Density}

In Study 2, we vary the number of nodes in the simulation area between 50 and 200. Forty packets for broadcast are 


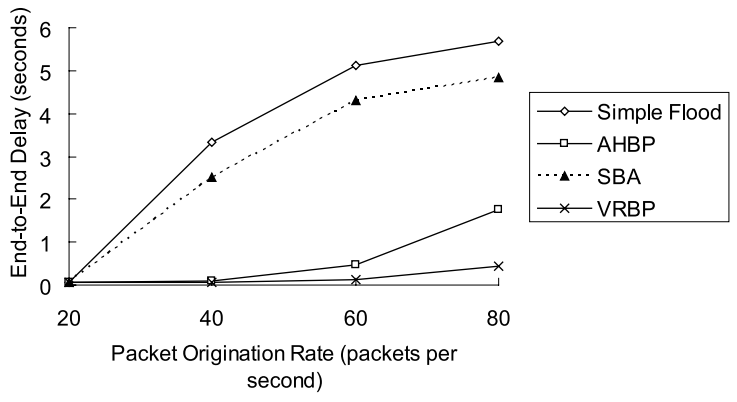

Fig. 4 End-to-end delay with respect to traffic load.

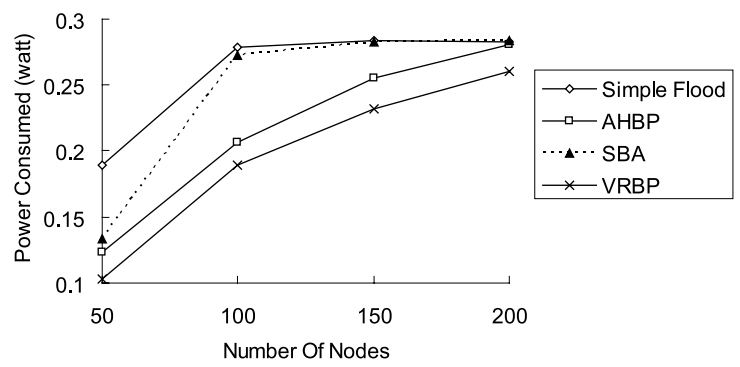

Fig. 5 Power consumption for various network density.

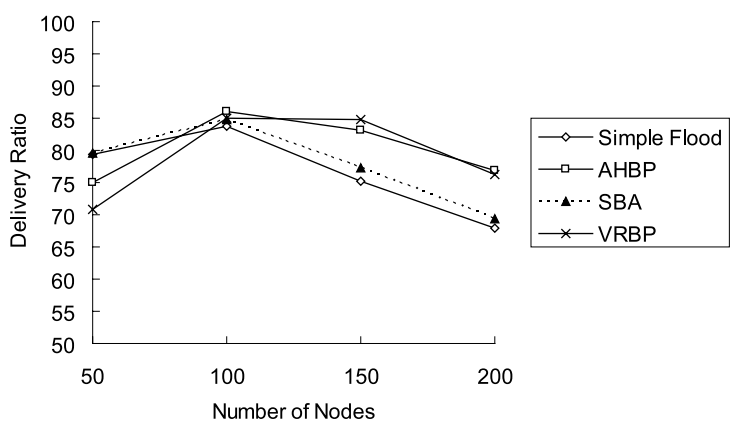

Fig. 6 Sensitivity of delivery ratio to network density.

generated in one second.

As the density increases, the network becomes more congested. As a result, collisions occur at a higher rate. By shortening the transmission range, the interference is alleviated and a high delivery ratio and a shorter end-to-end delay are obtained by VRBP (See Figs. 6 and 8). In Fig. 7, the number of rebroadcast nodes remains as low as that of AHBP.

Figure 5 shows that the total energy consumed by VRBP is lower than all of the common-range approaches considered herein. Note that the percentage of power savings for VRBP decreases when the network density is higher.

\section{Conclusion}

In this letter, we have presented a localized broadcast scheme using variable-range transmission power control. The present results indicate that the proposed variable-range approach can outperform other common-range transmission

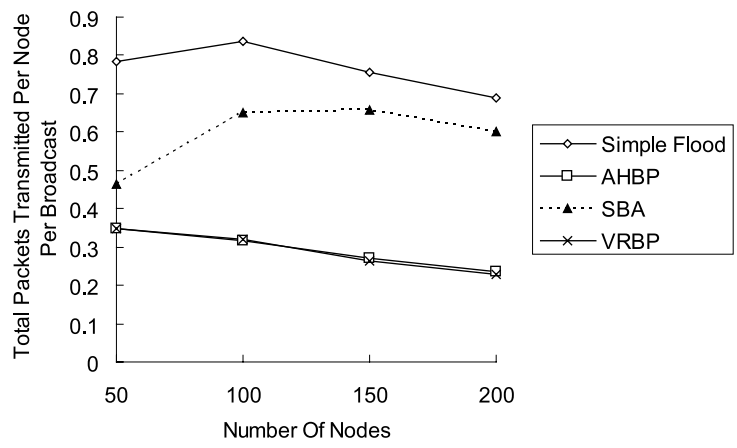

Fig. 7 Relationship between the ratio of rebroadcast nodes and the network density.

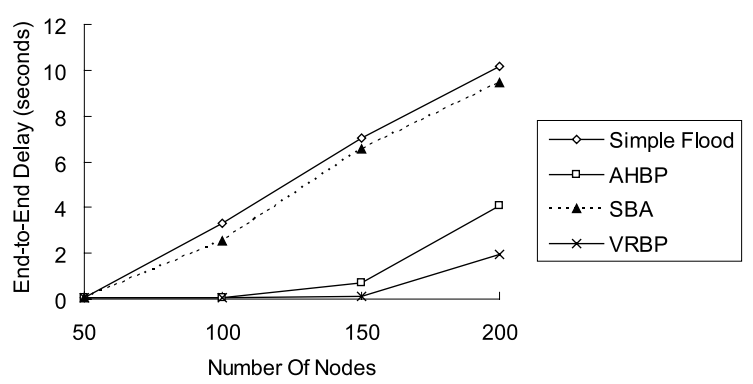

Fig. 8 End-to-end delay with respect to network density.

schemes in terms of power savings, and has increased capacity due to less interference and lower latency for message delivery. The simulation results also show that the end-toend delay of VRBP is the lowest regardless of the traffic load. This finding indicates that VRBP is a viable option for real-time multimedia broadcasting, because such broadcasting usually requires significant amounts of bandwidth, and a lower end-to-end delay helps to reduce the number of packets discarded due to violation of the timing constraints imposed.

In the future, we intend to compare the performance of VRBP with two existing schemes, [13] and [14]. There is also a need to simulate the proposed scheme on mobile networks in order to investigate its adaptivity to mobility.

\section{References}

[1] S. Ni, Y. Tseng, Y. Chen, and J. Sheu, "The broadcast storm problem in a mobile ad hoc network," Proc. ACM/IEEE International Conference on Mobile Computing and Networking (MOBICOM), pp.151-162, 1999.

[2] I. Stojmenovic and J. Wu, Mobile Ad Hoc Networking, ch. 7, pp.205-229, Wiley Interscience, 2004.

[3] Z. Haas, J. Halpern, and L. Li, "Gossip-based ad hoc routing," INFOCOM, pp.1707-1716, June 2002.

[4] J. Wu and F. Dai, "Broadcasting in ad hoc networks based on selfpruning," International Journal of Foundations of Computer Science, vol.2, no.12, pp.201-221, April 2003.

[5] I. Stojmenovic, M. Seddigh, and J. Zunic, "Dominating sets and neighbor elimination based broadcasting algorithms in wireless networks," IEEE Trans. Parallel Disrib. Syst., vol.1, no.13, pp.14-25, Jan. 2002.

[6] J. Susec and I. Marsic, "An efficient distributed network-wide broad- 
cast algorithm for mobile ad hoc networks," Technical Report, Rutgers University, 2000.

[7] W. Peng and X. Lu, "On reduction of broadcast redundancy in mobile ad hoc networks," Proc. MOBIHOC, pp.129-130, 2000.

[8] J. Wu and H. Li, "On calculating connected dominating set for efficient routing in ad hoc wireless networks," Proc. DiaLM, pp.7-14, 1999.

[9] A. Qayyum, L. Viennot, and A. Laouiti, "Multipoint relaying: An efficient technique for flooding in mobile wireless networks," Technical Report 3898, INRIA-Rapport de Recherche, 2000.

[10] W. Peng and X. Lu, "Ahbp: An efficient broadcast protocol for mobile ad hoc networks," Journal of Science and Technology, Beijing, China, vol.16, no.2, pp.114-125, 2001.

[11] B. William and T. Camp, "Comparison of broadcasting techniques for mobile ad hoc networks," Proc. MobiHoc, pp.194-205, June 2002.

[12] J. Wieselthier, G. Nguyen, and A. Ephremides, "On the construction of energy-efficient broadcast and multicast trees in wireless networks," Proc. IEEE INFOCOM, pp.585-594, 2000.

[13] J. Cartigny, D. Simplot, and I. Stojmenovic, "Localized minimumenergy broadcasting in ad hoc networks," Proc. IEEE INFOCOM, pp.2210-2217, 2003.

[14] X. Chen, M. Faloutous, and S. Krishnamurthy, "Power adaptive broadcasting with local information in ad hoc networks," Proc. IEEE ICNP, pp.168-178, 2003.

[15] K. Fall and K. Varadhan, The ns manual. Available at http://www.isi.edu/nsnam/ns/ 